\title{
An Unusual Complication During Bronchoscopy: Hypotension, Global ST Segment Elevation, and Acute Severe Left Ventricular Systolic Dysfunction
}

\author{
Sudeep Kumar DM, Alok Nath MD DM, Satyajeet Singh MD, \\ Tanuj Bhatia MD, and Aditya Kapoor DM
}

\begin{abstract}
Bronchoscopy is widely performed and generally considered safe. Cardiac complications during bronchoscopy are uncommon, and usually occur in elderly patients with coexistent coronary artery disease, hypertension, or severely impaired pulmonary function and resting hypoxemia. We report a patient who developed sudden onset restlessness, chest discomfort, hypotension, global ST elevation in multiple electrocardiogram leads, and acute severe left ventricular systolic dysfunction during a bronchoscopic transbronchial lymph node biopsy. Differential diagnosis included a massive myocardial infarction, apical ballooning (Tako-tsubo syndrome), or coronary vasospasm. The electrocardiogram changes resolved spontaneously, and a coronary angiogram 48 hours later revealed normal coronary artery anatomy and normal $\mathrm{LV}$ function. The patient made an uneventful recovery. It is important for physicians to be aware of such unusual complications to be able to appropriately manage these patients in clinical practice. Key words: bronchoscopy; cardiac; myocardial infarction. [Respir Care 2013;58(9):e111-e115. (C 2013 Daedalus Enterprises]
\end{abstract}

\section{Introduction}

The morbidity and mortality associated with bronchoscopy is low $(0.01-0.02 \%)$ and cardiovascular complications are extremely rare. ${ }^{1,2}$ We report a patient who developed sudden-onset chest discomfort, hypotension, ST segment elevation in multiple electrocardiogram (ECG) leads, and acute severe left ventricular (LV) systolic dysfunction during a bronchoscopic transbronchial lymph node biopsy. The ECG changes and cardiac biomarker assay indicated a massive ST elevation myocardial infarction. There was spontaneous resolution of the ECG changes,

The authors are affiliated with the Department of Cardiology, with the exception of Dr Nath, who is affiliated with the Department of Pulmonology, Sanjay Gandhi Postgraduate Institute of Medical Sciences, Lucknow, India.

The authors have disclosed no conflicts of interest.

Correspondence:: Aditya Kapoor DM, Department of Cardiology, Sanjay Gandhi Postgraduate Institute of Medical Sciences, Lucknow 226014, India. E-mail: akapoor65@gmail.com.

DOI: $10.4187 /$ respcare.02318 raising the possibility of "acute malignant coronary vasospasm" or apical ballooning (Tako-tsubo syndrome) as the underlying mechanism. A coronary angiogram 48 hours later revealed normal coronary artery anatomy and normal LV function, without any residual regional wall motion abnormalities. The patient was managed conservatively and made an uneventful recovery.

\section{Case Report}

The patient was a 61-year-old, non-diabetic, nonhypertensive, non-smoker, female who presented with gradually progressive shortness of breath and dry, nonproductive cough for the last 1 year. There was no history of fever, weight loss, or hemoptysis. She denied any history of angina or a cardiac event in the past. Routine hemogram and blood chemistry were normal, while a Mantoux test was strongly positive $(25 \mathrm{~mm}$ induration $48 \mathrm{~h}$ after purified protein derivative injection). Chest $\mathrm{x}$-ray revealed bilateral reticulonodular opacities, and computed tomography showed interlobular septal thickening, multiple area of bronchiectasis with cystic changes in the right middle lobe, multiple enlarged mediastinal lymph nodes, and bilateral hilar lymphadenopathy. During transbron- 

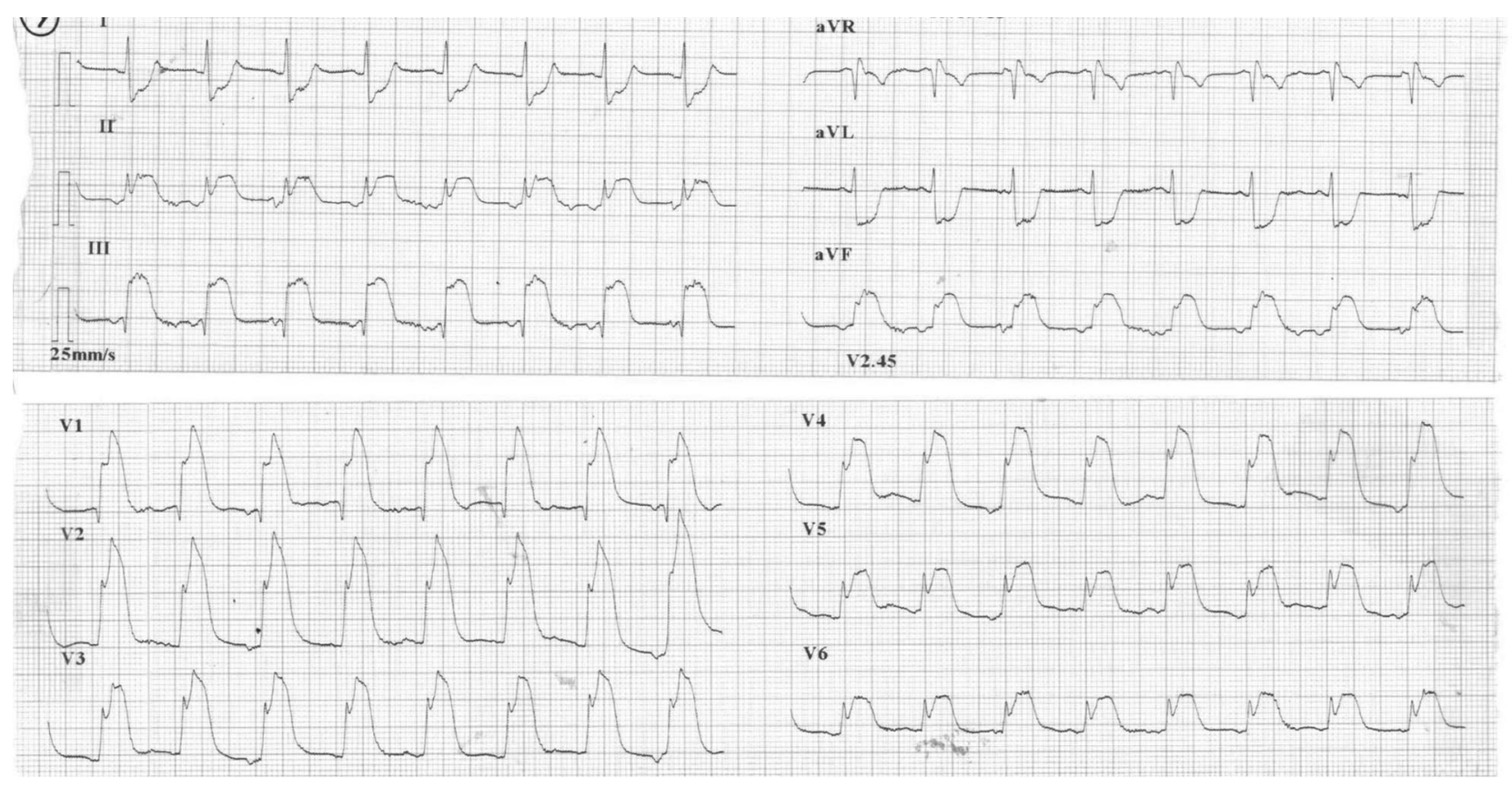

Fig. 1. Gross ST elevation on the inferior and anterior precordial leads of the 12-lead electrocardiogram.

chial lymph node biopsy to diagnose the lymphadenopathy in the right hilar lymph node region, the patient developed sudden restlessness, retrosternal chest discomfort, and a feeling of uneasiness. The ECG monitor revealed gross ST elevation, and the patient rapidly became diaphoretic, hypotensive, and hypoxemic. The procedure was immediately terminated, and efforts were instituted to maintain her blood pressure with intravenous fluid infusion and inotropic support. She was transferred to the ICU, where a 12 lead ECG revealed gross ST elevation on the inferior and anterior precordial leads (Fig. 1), suggestive of acute ST elevation myocardial infarction, and her blood pressure was $70 / 55 \mathrm{~mm} \mathrm{Hg}$.

In view of the ECG changes and likelihood of massive, acute myocardial infarction, a cardiology opinion was sought for consideration of transfer to the cardiac catheterization laboratory and urgent coronary angiography. A chest x-ray revealed no evidence of pneumothorax or pneumomediastinum. At the time of examination by the cardiology team she was conscious, oriented, and denied any ongoing chest pain. Another 12 lead ECG at this time interestingly revealed slight reduction in the ST elevation, especially in the inferior leads and precordial leads V4-V6 (Fig. 2).

An urgent bedside echocardiography revealed severe global hypokinesia and an overall LV ejection fraction of $30 \%$. Although a peri-procedural myocardial infarction was still the most likely possibility, in view of the spontaneous reduction in the ST segment elevation, a likelihood of "malignant coronary vasospasm" was also now considered. While the cardiac catheterization laboratory team was on standby, it was decided to perform serial ECGs and observe the temporal pattern of ST segment evolution before performing an urgent coronary angiogram. As the ST segment elevation gradually resolved, a brief run of rapid ventricular tachycardia was noted (Fig. 3), which was managed successfully with a bolus of amiodarone (150 mg intravenous). Serial ECGs over the next 30 min revealed progressive resolution of the ST segment elevation and normalization of the ST segment (Fig. 4)

Cardiac biomarker assay expectedly revealed elevated troponin-I $(9.2 \mathrm{ng} / \mathrm{mL})$ and creatine kinase-myocardial band (CKMB) $(12 \mathrm{ng} / \mathrm{mL})$, which further escalated to $80 \mathrm{ng} / \mathrm{mL}$ and $178 \mathrm{ng} / \mathrm{mL}$, respectively, at 6 hours. She made a rapid, uneventful recovery over the next 48 hours. A coronary angiogram 48 hours later revealed normal coronary arteries (Fig. 5). The LV angiogram also revealed normal LV function, without any regional wall motion abnormalities (Fig. 6), therefore suggesting possible acute coronary vasospasm as the etiology of the global ST elevation.

\section{Discussion}

Cardiac complications, such as arrhythmia, myocardial ischemia, and infarction, during bronchoscopy are uncommon, and generally occur in elderly patients with comorbidities such as preexisting coronary artery disease, hypertension, or severe impairment of pulmonary function and resting hypoxemia. ${ }^{3,4}$ Various cardiac arrhythmias, ranging from atrial and ventricular premature complexes or supraventricular tachycardias to ventricular tachycar- 


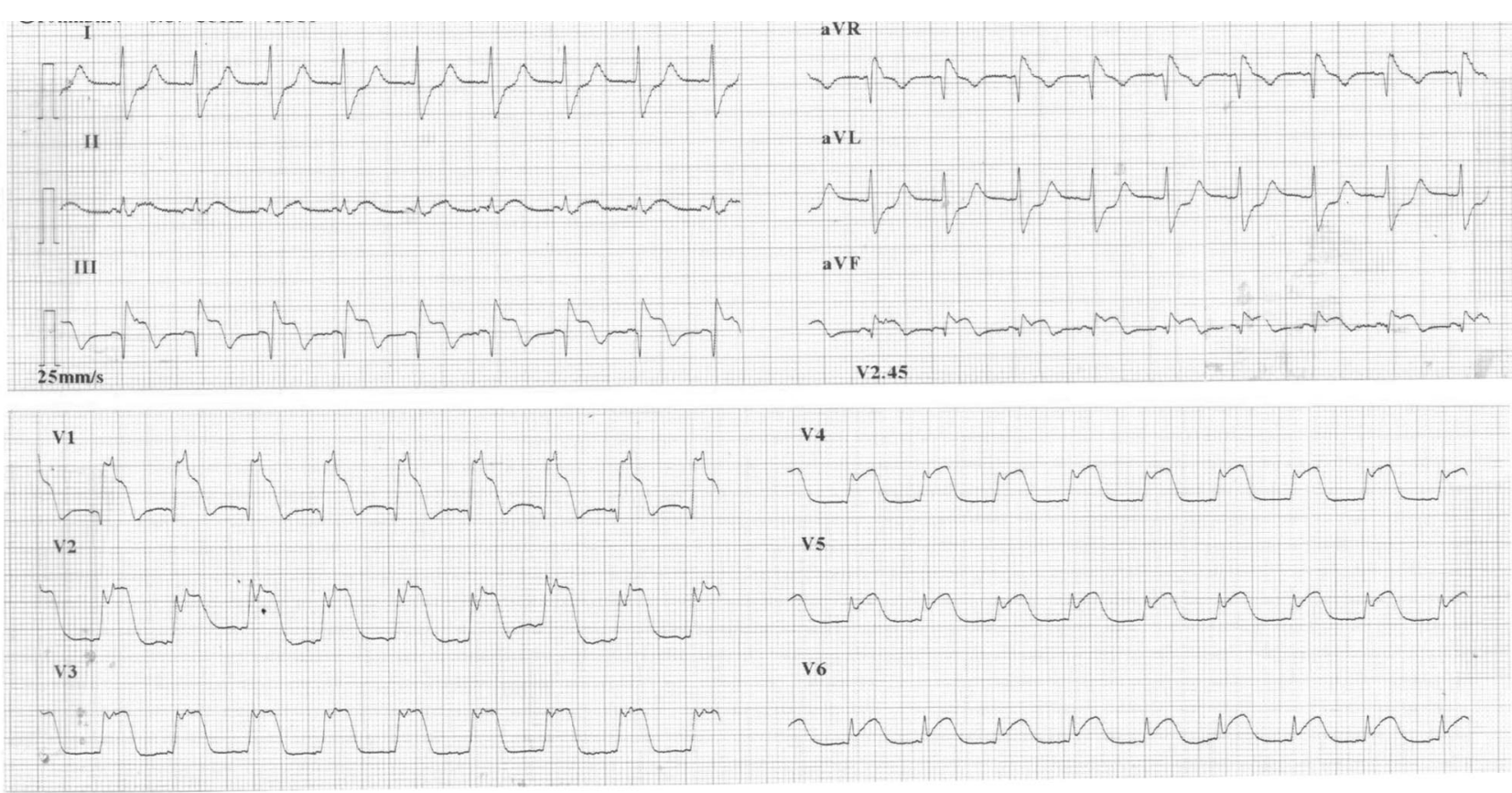

Fig. 2. Second 12-lead electrocardiogram reveals slight reduction in the ST elevation, especially on the inferior leads and precordial leads V4-V6.

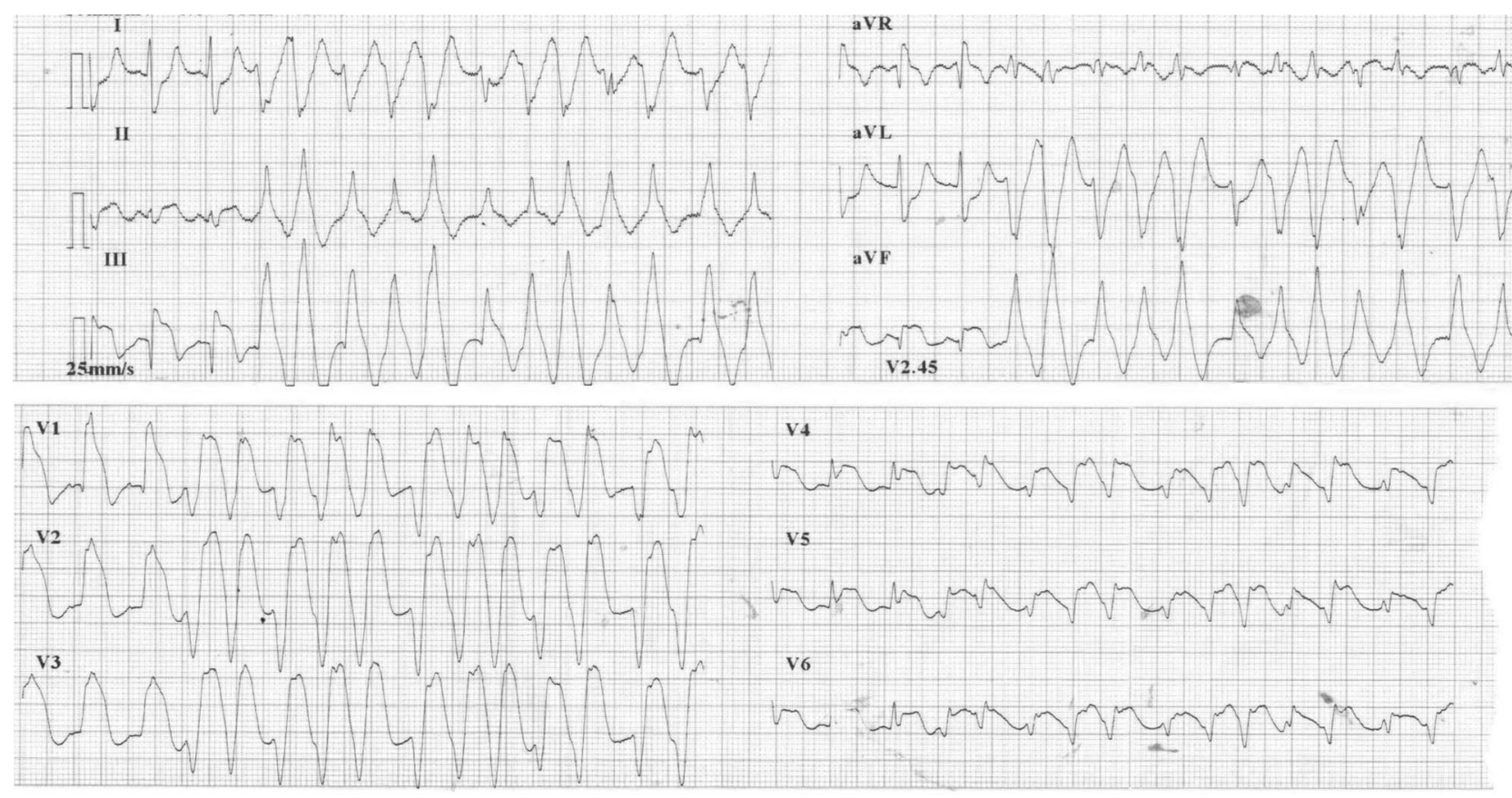

Fig. 3. A brief run of rapid ventricular tachycardia during the resolution phase of the ST elevation.

dias, bradyarrhythmias, and even cardiac arrest, have been reported during bronchoscopy.,2,-7 Myocardial ischemia and acute ST elevation myocardial infarction following bronchoscopy, although uncommon, have also been reported. ${ }^{1,8,9}$
Cardiac ischemia and arrhythmias, although rare, are generally due to elevated circulating catecholamines, precipitation of true myocardial ischemia, or following administration of anesthetic drugs, especially in patients with underlying hypoxemia and other comorbidities. 


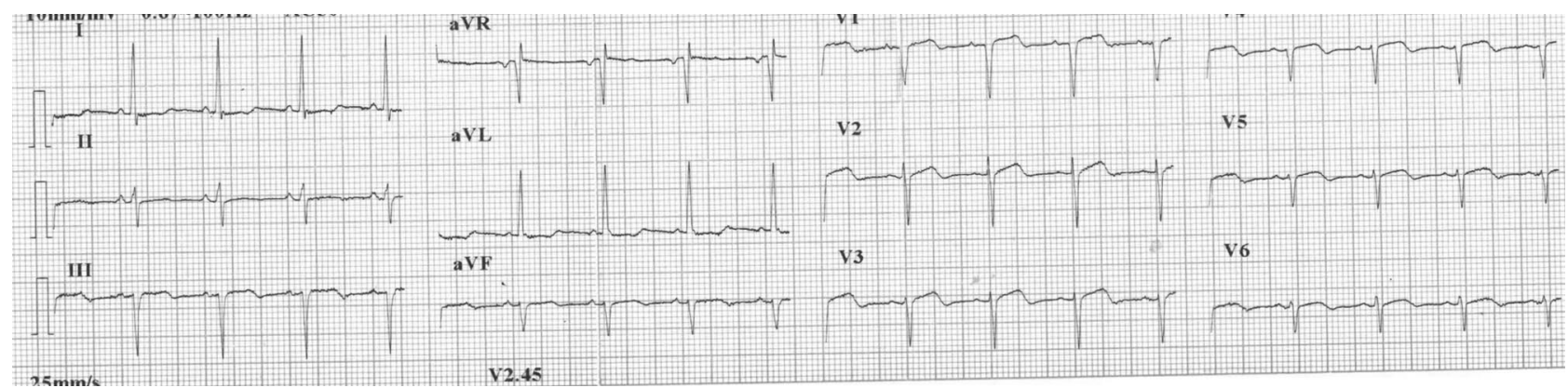

Fig. 4. Progressive resolution of the ST segment elevation and normalization of the ST segment.

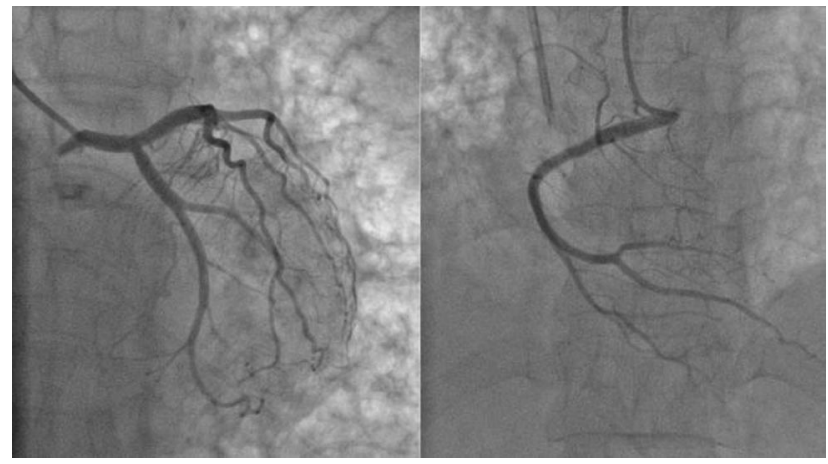

Fig. 5. Coronary angiogram reveals normal coronary arteries. Left: Left coronary artery. Right: Right coronary artery.

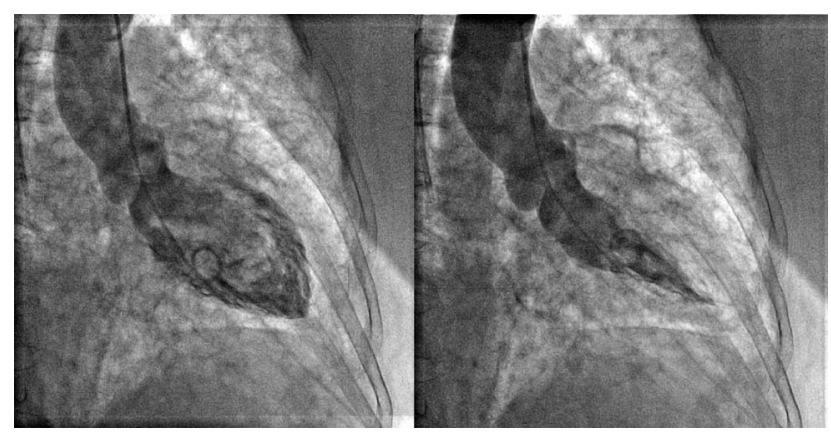

Fig. 6. Left ventricular angiogram shows normal left ventricular function, with no regional wall motion abnormalities. Left: Diastolic frame. Right: Systolic frame.

The ECG changes in our patient suggested a massive myocardial infarction, which was corroborated by the elevated cardiac biomarker levels. We initially planned an urgent coronary angiogram (in view of the ECG findings, hypotension, and echocardiographic evidence of LV dysfunction), but because of the spontaneous resolution of the ECG changes (in absence of any fibrinolysis or coronary intervention), the coronary angiogram was deferred, and serial ECGs revealed complete normalization of the ST changes. Had the ECGs not shown this spontaneous resolution or had her chest pain continued, urgent coronary angiogram would definitely have been performed, and the cardiac catheterization laboratory team was on continuous standby.

The pathogenesis was believed to be either apical ballooning (Tako-tsubo syndrome) or "acute malignant coronary vasospasm." Apical ballooning is characterized by sudden, severe, transient, segmental dysfunction of the LV due to a hyper-adrenergic state, usually following intense emotional or physical stress. The clinical presentation simulates that of an acute myocardial infarction, with ST elevation in the ECG and rapid development of acute LV systolic dysfunction. ${ }^{10,11}$ In these patients coronary angiography typically reveals normal coronary anatomy and impaired LV systolic function (apical ballooning), which rapidly resolves, without any residual impairment. Guerrero et al recently reported the occurrence of Tako-tsubo syndrome following bronchoscopy. ${ }^{12}$ However, in contrast to our patient, their patient had no important ECG changes or troponin rise, although coronary angiography confirmed normal coronary anatomy with severe LV dysfunction.

Due to the presence of the completely normal LV function, without any regional wall motion abnormality on the LV angiogram, we hypothesized that "acute malignant coronary vasospasm" could be the underlying pathophysiology of the global ST elevation in our patient. The diffuse coronary vasospasm may have resulted in global myocardial ischemia, ST elevation, and acute severe LV systolic dysfunction. It is possible that our patient had Tako-tsubo syndrome and the LV function had completely recovered by the time of the coronary angiography. However, the LV was initially globally hypokinetic in our patient, while in cases of apical ballooning syndrome, apical akinesia or dyskinesia with preserved or hyperkinetic contractile basal LV segments are more typical.

This case highlights the occurrence of an extensive pseudo-myocardial infarction pattern as a complication of bronchoscopy, in a patient without underlying coronary artery disease, possibly secondary to "acute malignant coronary vasospasm." The case highlights the fact that such life-threatening complications can occur during bronchoscopy. Since it is not possible to predict the occurrence of such complications, routine ECG (especially for elderly/ 


\section{An Unusual Complication During Bronchoscopy}

high-risk patients) during bronchoscopy is important for early diagnosis and appropriate management of such adverse effects.

\section{REFERENCES}

1. Credle WF Jr, Smiddy JF, Elliot RC. Complications of fiberoptic bronchoscopy. Am Rev Respir Dis 1974;109(1):67-72.

2. Surratt PM, Smiddy JF, Gruber B. Deaths and complications associated with fiberoptic bronchoscopy. Chest 1976;69(6):747-751.

3. Cavaliere S, Foccoli P, Farina PL. Nd:YAG Laser bronchoscopy. A five-year experience with 1,396 applications in 1,000 patients. Chest 1988;94(1):15-21.

4. Davies L, Mister R, Spence DP, Calverley PM, Earis JE, Pearson MG. Cardiovascular consequences of fibreoptic bronchoscopy. Eur Respir J 1997;10(3):695-698.

5. D'Aloia A, Faggiano P, Fiorina C, Vizzardi E, Cavaliere S, Foccoli $\mathrm{P}$, et al. Cardiac arrest due to ventricular fibrillation as a complication occurring during rigid bronchoscopic laser therapy. Monaldi Arch Chest Dis 2003;59(1):88-90.

6. Elguindi AS, Harrison GN, Abdulla AM, Chaudhary BA, Vallner JJ, Kolbeck RC, Speir WA Jr. Cardiac rhythm disturbances during fi- beroptic bronchoscopy: a prospective study. J Thorac Cardiovasc Surg 1979;77(4):557-561.

7. Katz AS, Michelson EL, Stawicki J, Holford FD. Cardiac arrythmias: frequency during fiberoptic bronchoscopy and correlation with hypoxemia. Arch Intern Med 1981;141(5):603-606.

8. Matot I, Drenger B, Glantz L, Kramer MR. Coronary spasm during outpatient fiberoptic laser bronchoscopy. Chest 1999;115(6):17441746.

9. Osula S, Stockton P, Abdelaziz MM, Walshaw MJ. Intratracheal cocaine induced myocardial infarction: an unusual complication of fibreoptic bronchoscopy. Thorax 2003;58(8):733-734.

10. Gianni M, Dentali F, Grandi AM, Sumner G, Hiralal R, Lonn E. Apical ballooning syndrome or takotsubo cardiomyopathy: a systematic review. Eur Heart J 2006;27(13):1523-1529.

11. Kurowski V, Kaiser A, von Hof K, Killermann DP, Mayer B, Hartmann F. Apical and midventricular transient left ventricular dysfunction syndrome (Tako-tsubo cardiomyopathy): frequency, mechanisms, and prognosis. Chest 2007;132(3):809-816.

12. Guerrero J, Majid A, Ernst A. Cardiogenic shock secondary to Takotsubo syndrome after debridement of malignant endobronchial obstruction. Chest 2009;135(1):217-220. 